\title{
Status and Correlates of Hypersomnia in Hospitalized Patients with Unipolar Depression - Beijing, Henan, and Shandong, China, August 2019-March 2021
}

\author{
Yun Chen ${ }^{1, x}$; Lyufeng Zhang ${ }^{2, x ;}$ Sifan $\mathrm{Hu}^{1, * ;}$; Hongle Zhang ${ }^{3}$; Qiqing Sun ${ }^{1}$; \\ Mengyue Hong'; Yuying Qian ${ }^{2}$; Lin Lu'; Hongqiang Sun ${ }^{1, t}$
}

\section{Summary \\ What is already known about this topic? \\ Hypersomnia is an atypical characteristic of unipolar depression (UD), indicating a high risk of bipolar depression. Identifying the symptom should be prioritized in patients with UD. However, the status and correlated factors of hypersomnia greatly varied across investigations. \\ What is added by this report? \\ Among inpatients with UD, the rate of hypersomnia was roughly $28.1 \%$ (39/139). Younger age (18-35 years) and recurrent depression were independent correlates of hypersomnia in hospitalized patients with UD. \\ What are the implications for public health practice? \\ Understanding the high rate and independent correlates of hypersomnia in hospitalized patients with UD will help clinicians and policymakers to identify characteristics of depression, strengthen the management capacity and improve the quality of treatment and control programs.}

Depression remains a serious public health problem around the world, including China. Globally, depression accounts for the biggest share of the total burden of disease with an estimated 76.4 million years lost to the disability (1). According to the data from the China Mental Health Survey, which was launched nationally in 2012, depressive disorder was the most common mood disorder, and its weighted lifetime prevalence was up to $6.8 \%$ (2), thus bringing heavy disease burden in Chinese population. Previously, the clinical and health management of unipolar depression (UD) emphasized symptoms of mood and interest but ignored the recognition of sleep disturbance, especially hypersomnia. Hypersomnia is an atypical characteristic of depressive disorder and it is significant for the early identification of bipolar depression from UD (3). However, the rate and correlates of hypersomnia greatly varied across investigations. In the study, we investigated hospitalized patients with UD from 3 hospitals in Beijing Municipality, Henan Province, and Shandong Province and found that the rate of hypersomnia was roughly $28.1 \%$ (39/139), and younger age (18-35 years) and recurrent depression were independent correlated factors of hypersomnia in hospitalized patients with UD. The result reminded us of the necessity of paying attention to hypersomnia in UD patients.

The investigation was a cross-sectional study, and a total number of 139 inpatients with UD were recruited from Peking University Sixth Hospital, Zhumadian Second People's Hospital, and Liaocheng Fourth People's Hospital from August 2019 to March 2021. The research protocol was approved by the Ethics Committee of Peking University Sixth Hospital, and all participants signed informed consent. All inpatients met the unipolar depressive disorder diagnostic criteria of the International Classification of Diseases-10th Version (ICD-10) and were confirmed by the MiniInternational Neuropsychiatric Interview (M.I.N.I.). They were all 18 years old or older and had primary education or above. All inpatients scored more than or equal to 20 in the Mini Mental State Examination. Patients with serious impairment of cardiac, hepatic, nephritic, and respiratory function or other serious diseases were excluded from the study. The general demographic data and clinical characteristics were obtained, including the Epworth Sleepiness Scale (ESS), the 9-item Patient Health Questionnaire (PHQ-9), the 7-item Generalized Anxiety Disorder Scale (GAD-7), the Brief Psychiatric Rating Scale (BPRS), and the Montreal Cognitive Assessment (MoCA); the suicide module of M.I.N.I. Hypersomnia was determined by a score of more than or equal to 10 in ESS (4) (total score range is between 1-24), all 
participants were divided into groups of inpatients with and without hypersomnia depending on the scores of ESS.

Measurement data of normal distribution were expressed as $\bar{x} \pm s$, and an independent sample t-test was used for comparison between the two groups. Measurement data of non-normal distribution was represented by $M\left(\mathrm{Q}_{1}, \mathrm{Q}_{3}\right)$, and the Mann-Whitney $\mathrm{U}$ test was used for comparison between the two groups. Count data were expressed as $\mathrm{n}(\%)$, and $\chi^{2}$ test was used for comparison. A multiple logistic regression model was used to obtain the independent correlates of hypersomnia. All statistical methods were adopted by the two-tailed test, and $P<0.05$ was considered statistically significant. All statistical analyses were performed using SPSS software (Version 21.0. IBM Corp., Armonk, NY, USA).

Among all hospitalized patients with UD, $41.7 \%$ (58/139) of participants were male and $58.3 \%$ $(81 / 139)$ were female. The age of all the patients had a mean value of $43.45 \pm 16.37$ years old. The rate of hypersomnia in hospitalized patients with UD was roughly $28.1 \%$ (39/139), scoring more than or equal to 10 in ESS. The rate of recurrent depression in the UD inpatients was 46.8\% (65/139). Depression above the threshold was defined by a score of more than or equal to 10 in PHQ-9 (5), and its rate was $71.2 \%$ (99/139).

In terms of demographic characteristics, compared with inpatients without hypersomnia, inpatients with hypersomnia were younger, aged with a median value of 31 years $(Z=-3.77, P<0.001)$. After stratifying by age, inpatients with hypersomnia also had high rate of younger age (18-35 years) $(66.7 \%$ vs. $28.0 \%$, $P<0.001)$. Gender distribution had no significant difference between two groups. (Table 1)

For the clinical characteristics, there were no significant differences in the duration of depression, family history of mental disorders, history of modified electroconvulsive therapy (MECT), histories of smoking and alcohol consumption, and use of medications between UD inpatients with and without hypersomnia. The hypersomnia group had a higher rate of recurrent depression, but the difference was not statistically significant $(59.0 \%$ vs. $42.0 \%, P=0.072)$. Compared with participants without hypersomnia, those with hypersomnia had higher scores of MoCA. However, the rate of mild cognitive impairment, defined by the score of less than 26 in MoCA, showed no significant difference between the two groups. Also, there were no significant differences in BPRS scores,
GAD-7 scores, and rates of comorbidity with anxiety and current suicide risk between inpatients with and without hypersomnia. UD inpatients with hypersomnia scored significantly higher in PHQ-9 than those without hypersomnia $(P<0.05)$, indicating severer depressive symptoms. Patients with hypersomnia had a higher rate of depression above the threshold, but the statistical difference was not significant $(82.1 \%$ vs. $67.0 \%, P=0.078)$.

In the multiple logistic regression model, age, recurrent depression, and depression above the threshold (PHQ-9 $\geq 10$ ) were entered as potential contributors to hypersomnia in hospitalized patients with UD. As presented in Table 2, being aged 18-35 years [odds ratio $(\mathrm{OR})=6.73,95 \%$ confident interval (CI): 1.91-23.75] and recurrent depression $(\mathrm{OR}=2.96$, 95\% CI: 1.25-6.99) were independently correlated with hypersomnia in hospitalized patients with UD (all $P<0.05)$. However, the factor of depression above the threshold was not an independent correlate of hypersomnia.

\section{DISCUSSION}

The study was a multicenter cross-sectional epidemiological investigation that found that the rate of hypersomnia in UD inpatients was roughly $28.1 \%$, and patients with hypersomnia were associated with severer depressive symptoms. Younger age (1835 years) and recurrent depression were independently associated with hypersomnia.

A previous study had reported the prevalence of increased sleep in major depressive disorder was $24.0 \%$ (3), which was similar to the rate of hypersomnia in hospitalized patients with UD. After controlling the effect of medication types, our study revealed younger age (18-35 years) and recurrent depression were independent correlates of hypersomnia in patients with UD. Several studies have illustrated that atypical depression might serve as a nosological bridge between UD and type II bipolar depression and indicate the possibility of "soft bipolarity". Bipolar depression is so similar to UD in its clinical manifestation that often obscures the differential diagnosis and accurate recognition. Clinical misdiagnosis may lead to incorrect intervention and poor prognosis, such as iatrogenic mania/hypomania switching and higher suicide risk, and thus higher healthcare burdens for individuals and the government. Bipolar disorders brought heavy disease burden worldwide and are ranked as being a heavier health burden in younger 
TABLE 1. Comparisons of demographic and clinical characteristics in unipolar depressive inpatients with and without hypersomnia.

\begin{tabular}{|c|c|c|c|c|}
\hline Characteristics & $\begin{array}{c}\text { Inpatients with } \\
\text { hypersomnia }(n=39)\end{array}$ & $\begin{array}{c}\text { Inpatients without } \\
\text { hypersomnia }(n=100)\end{array}$ & $t / X^{2} / Z$ value & $P$ value \\
\hline \multicolumn{5}{|l|}{ Demographic characteristics } \\
\hline Gender [Male/Female, n (\%)] & $17 / 22(43.6 / 56.4)$ & $41 / 59(41.0 / 59.0)$ & 0.08 & 0.781 \\
\hline Age $\left(\right.$ Years, $\left.M\left(Q_{1}, Q_{3}\right)\right]$ & $31.00(22.00,47.00)$ & $51.00(34.00,57.75)$ & -3.77 & $<0.001$ \\
\hline $18-35$ years $[n(\%)]$ & $26(66.7)$ & $28(28.0)$ & & \\
\hline $36-59$ years $[\mathrm{n}(\%)]$ & $9(23.1 \%)$ & $51(51.0)$ & 17.67 & $<0.001$ \\
\hline$>60$ years $[n(\%)]$ & $4(10.3 \%)$ & $21(21.0)$ & & \\
\hline \multicolumn{5}{|l|}{ Clinical characteristics } \\
\hline Duration of depression (Years, $\left.M\left(Q_{1}, Q_{3}\right)\right]$ & $3.00(1.00,9.75)$ & $3.00(1.00,10.00)$ & -0.56 & 0.578 \\
\hline Recurrent depression [n (\%)] & $23(59.0)$ & $42(42.0)$ & 3.25 & 0.072 \\
\hline Family history of mental disorders [n (\%)] & $7(17.9)$ & $26(26.0)$ & 1.01 & 0.316 \\
\hline History of alcohol consumption [n (\%)] & $36(92.3)$ & $83(83.0)$ & 1.97 & 0.160 \\
\hline History of smoking [n (\%)] & $33(84.6)$ & $75(75.0)$ & 1.50 & 0.221 \\
\hline History of MECT [n (\%)] & $35(89.7)$ & $82(82.0)$ & 1.26 & 0.261 \\
\hline \multicolumn{5}{|l|}{ Medication [n (\%)] } \\
\hline Antipsychotics & $22(56.4)$ & $46(46.0)$ & 1.22 & 0.270 \\
\hline Antidepressants & $39(100)$ & $98(98.0)$ & 0.79 & 0.374 \\
\hline Sedative hypnotics & $27(69.2)$ & $69(69.0)$ & 0.001 & 0.979 \\
\hline Mood Stabilizers & $2(5.1)$ & $5(5.0)$ & 0.001 & 0.975 \\
\hline MoCA score $\left[M\left(Q_{1}, Q_{3}\right)\right]$ & $25.00(22.00,28.00)$ & $23.00(20.00,26.75)$ & -1.97 & 0.049 \\
\hline Mild cognitive impairment (MoCA score<26) [n (\%)] & $23(59.0)$ & $66(66.0)$ & 0.60 & 0.438 \\
\hline BPRS score $(\bar{x} \pm s)$ & $32.46 \pm 6.68$ & $32.57 \pm 5.27$ & 0.10 & 0.920 \\
\hline PHQ-9 score $\left[M\left(Q_{1}, Q_{3}\right)\right]$ & $16.00(12.00,23.00)$ & $13.00(8.00,20.00)$ & -1.99 & 0.047 \\
\hline Depression (PHQ-9 score $\geq 10)$ [n (\%)] & $32(82.1)$ & $67(67.0)$ & 3.10 & 0.078 \\
\hline GAD-7 score $\left[M\left(Q_{1}, Q_{3}\right)\right]$ & $11.00(6.00,16.00)$ & $8.00(5.00,14.00)$ & -1.45 & 0.149 \\
\hline Anxiety (GAD-7 score $\geq 10)[n(\%)]$ & $22(56.4)$ & $42(42.0)$ & 2.35 & 0.126 \\
\hline Current suicide risk [n (\%)] & $30(76.9)$ & $68(68.0)$ & 1.07 & 0.300 \\
\hline
\end{tabular}

Abbreviations: MECT=modified electroconvulsive therapy; MoCA=montreal cognitive assessment; $B P R S=$ brief psychiatric rating scale; $\mathrm{PHQ}-9=$ 9-item patient health questionnaire; GAD-7=7-item generalized anxiety disorder scale.

TABLE 2. Independent correlates of hypersomnia in hospitalized unipolar depressive patients ( $n=139)$.

\begin{tabular}{|c|c|c|c|c|c|}
\hline Items & B value & S.E. value & Wald $x^{2}$ value & OR (95\% Cl) & $P$ value \\
\hline \multicolumn{6}{|l|}{ Age (years) } \\
\hline$>60$ & & & & 1 & \\
\hline $36-59$ & 0.041 & 0.666 & 0.004 & $1.042(0.282-3.844)$ & 0.951 \\
\hline $18-35$ & 1.906 & 0.644 & 8.766 & 6.726 (1.905-23.751) & 0.003 \\
\hline \multicolumn{6}{|c|}{ Recurrent depression } \\
\hline No & & & & 1 & \\
\hline Yes & 1.084 & 0.439 & 6.102 & $2.956(1.251-6.987)$ & 0.014 \\
\hline
\end{tabular}

Abbreviations: S.E.=standard erro; OR=odds ratio; $\mathrm{Cl}=$ Confidence interval.

populations (G), which may be a consequence of the early age of onset and recurrent nature of bipolar disorder ( 7 ). Therefore, it can be inferred that UD patients with hypersomnia have some inherent characteristics that may be associated with bipolar depression and may develop into bipolar disorder in 
the future. However, the mechanisms of the relationship between the two have not been clarified, and further longitudinal research and exploration are still needed.

This study was subject to at least three limitations. First, the cross-sectional study design could only present correlated factors of hypersomnia, and it is difficult to avoid recall bias and cannot reveal the influencing factors and causal relationships. Second, ESS only revealed the subjective sleep quality in the last several months, which cannot fully reflect the longterm circumstances and objective performance. Finally, the relatively small sample size limited the reliability of the study, so further investigations with larger sample sizes are necessary.

In conclusion, hypersomnia is common in hospitalized patients with UD. We should pay close attention to the evaluation of hypersomnia in UD to help guide clinical practice and health management.

Conflicts of interest: No conflicts of interest declared.

Acknowledgements: Project teams from Peking University Sixth Hospital, Zhumadian Second People's Hospital and Liaocheng Fourth People's Hospital, and all participants.

Funding: National Natural Science Foundation of China (81971235, 81771429); Fundamental Research Funds for the Central Universities, Peking University Medicine Fund B of Fostering Young Scholars' Scientific \& Technological Innovation (BMU2021 PYB045).

doi: $10.46234 / \mathrm{ccdcw} 2021.217$
\# Corresponding authors: Hongqiang Sun, sunhq@bjmu.edu.cn; Sifan Hu, husifan@bjmu.edu.cn.

${ }_{1}$ Peking University Sixth Hospital, Peking University Institute of Mental Health, NHC Key Laboratory of Mental Health, National Clinical Research Center for Mental Disorders (Peking University Sixth Hospital), National Center for Mental Health, Chinese Center for Disease Control and Prevention, Beijing, China; ${ }^{2}$ Zhumadian Second People's Hospital, Zhumadian Psychiatric Hospital, Zhumadian, Henan, China; ${ }^{3}$ Liaocheng Fourth People's Hospital, Liaocheng, Shandong, China.

\& Joint first authors.

Submitted: September 24, 2021; Accepted: October 12, 2021

\section{REFERENCES}

1. Smith K. Mental health: a world of depression. Nature 2014;515(7526): 180 - 1. http://dx.doi.org/10.1038/515180a.

2. Huang YQ, Wang Y, Wang H, Liu ZR, Yu X, Yan J, et al. Prevalence of mental disorders in China: a cross-sectional epidemiological study. Lancet Psychiat 2019;6(3):211 - 24. http://dx.doi.org/10.1016/s22150366(18)30511-x.

3. Akiskal HS, Benazzi F. Atypical depression: a variant of bipolar II or a bridge between unipolar and bipolar II? J Affect Disorders 2005;84 (2-3):209 - 17. http://dx.doi.org/10.1016/j.jad.2004.05.004.

4. Johns MW. A new method for measuring daytime sleepiness: the Epworth sleepiness scale. Sleep 1991;14(6):540 - 5. http://dx.doi.org/ 10.1093/sleep/14.6.540.

5. Levis B, Sun Y, He C, Wu Y, Krishnan A, Bhandari PM, et al. Accuracy of the PHQ-2 alone and in combination with the PHQ-9 for screening to detect major depression: systematic review and meta-analysis. JAMA 2020;323(22):2290 - 300. http://dx.doi.org/10.1001/jama.2020.6504.

6. Gore FM, Bloem PJN, Patton GC, Ferguson J, Joseph V, Coffey C, et al. Global burden of disease in young people aged 10-24 years: a systematic analysis. Lancet 2011;377(9783):2093 - 102. http://dx.doi.org/10. 1016/S0140-6736(11)60512-6.

7. Scott J, Morton E, Michalak EE, Murray G. An epidemiological study of externally determined and self-identified mental and physical disability across the spectrum of bipolar disorders. J Affect Disorders 2021;294: 597 - 604. http://dx.doi.org/10.1016/j.jad.2021.07.085. 